\title{
Combined effects of leaks, respiratory system properties and upper airway patency on the performance of home ventilators: a bench study
}

Kaixian Zhu ${ }^{1 *}$ (D), Claudio Rabec ${ }^{2}$, Jésus Gonzalez-Bermejo ${ }^{3,4}$, Sébastien Hardy ${ }^{1}$, Sami Aouf ${ }^{1}$, Pierre Escourrou ${ }^{5}$ and Gabriel Roisman ${ }^{5}$

\begin{abstract}
Background: Combined effects of leaks, mechanical property of respiratory system and upper airway (UA) patency on patient-ventilator synchrony (PVA) and the level of clinically "tolerable" leaks are not well established in home ventilators.

Methods: We comparatively assessed on a bench model, the highest leak level tolerated without inducing significant asynchrony ("critical leak") in three home ventilators (Astral 150, Trilogy 100 and Vivo 60; noted as A150, T100 and V60 respectively) subjected to three simulated diseased respiratory conditions: chronic obstructive pulmonary disease (COPD), obesity hypoventilation (OHS) and neuromuscular disorders (NMD), with both open and closed UA. Also, total leak values in the device reports were compared to the bench-measured values.

Results: With open UA, all ventilators were able to avoid asynchrony up to a $30 \mathrm{~L} / \mathrm{min}$ leak and even to $55 \mathrm{~L} / \mathrm{min}$ in some cases. UA closure and respiratory diseases especially OHS influenced PVA. With closed UA, the critical leak of A150 and T100 remained higher than $55 \mathrm{~L} / \mathrm{min}$ in COPD and OHS, while for V60 decreased to 41 and $33 \mathrm{~L} /$ min respectively. In NMD with closed UA, only T100 reached a high critical leak of $69 \mathrm{~L} / \mathrm{min}$. Besides, inspiratory trigger sensitivity change was often necessary to avoid PVA.
\end{abstract}

Conclusions: Home ventilators were able to avoid PVA in high-level leak conditions. However, asynchrony appeared in cases of abnormal mechanical properties of respiratory system or closed UA. In case of closed UA, the EPAP should be adjusted prior to the inspiratory trigger.

Trial registration: Not applicable.

Keywords: Non-invasive ventilation, Patient-ventilator asynchrony, Unintentional leak, Upper airway patency

\section{Background}

Home non-invasive ventilation (NIV) is a wellestablished treatment for patients with chronic hypercapnic respiratory failure so that the number of patients equipped with NIV devices has been steadily increasing all over the world [1-3]. To prevent the progression of sleep time toward daytime hypercapnia and further severe respiratory failure, home NIV is predominantly applied during sleep time.

\footnotetext{
* Correspondence: kaixian.zhu@gmail.com

${ }^{1}$ Centre Explor, Air Liquide Healthcare, 28 rue d'Arcueil, 94250 Gentilly, France

Full list of author information is available at the end of the article
}

There are two specific characteristics of NIV that affect the ventilation effectiveness, particularly during sleep. First, because NIV is delivered via a nasal or fullface mask, unintentional leak is a commonly found drawback as a consequence of the non-hermetic nature of the system [4-6]. Leakage may be absent or minimal when the patient is awake but often worsen during sleep as a result of the loss of voluntary control and decreased muscle tone. Second, under NIV, intermittent obstruction of the upper airway (UA) is commonly observed during sleep in subjects with increased UA collapsibility. In chronic NIV, both obstructive breathing events and 
unintentional leaks are known to induce the occurrence of patient-ventilator asynchronies (PVA), which may reduce both ventilator efficacy and sleep quality [7-9] and are associated with decreased patient survival $[10,11]$.

Adjustable inspiratory triggering which is more sensitive than classical flow triggering is supposed to reduce PVA. Moreover, with latest generation blowers, very high-level leaks can be compensated. Nevertheless, the level of leaks that can be tolerated by the NIV ventilators for maintaining patient-ventilator synchronization is unknown.

The goal of this study was to comparatively assess in a bench model, the maximal leak level tolerated without inducing significant asynchrony ("critical leak") in ventilators subjected to three diseased respiratory conditions: chronic obstructive pulmonary disease (COPD), obesity hypoventilation syndrome (OHS) and neuromuscular disorders (NMD). Each disease was simulated with both open and closed UAs.

\section{Methods}

\section{Bench model}

Evaluations were carried out on a bench set-up that has been modified from a previously described respiratory model [12]. The bench model consisted of an active lung ASL5000 (IngMar Medical, Pittsburgh, USA) and a Starling resistor. This system allows simulating physiological and pathological conditions of the respiratory system, by varying respiratory mechanics, inspiratory effort as well as UA patency. The modified bench model (Fig. 1) included a variable-opening valve located downstream to the calibrated intentional nasal leak port $(24 \mathrm{~L} / \mathrm{min}$ at 10 $\mathrm{cmH} 2 \mathrm{O}$ ) to simulate different levels of unintentional leak. The total leak was continuously measured by a pneumotachograph with digital display and analog output (Model 4040 Flowmeter, TSI Inc., MN, USA), which is linear in the studied leak flow ranges. A manometer (MecoSmart D-06, Mesureur, Chilly-Mazarin, France) was added to measure the pressure downstream to the Starling resistor (Tracheal pressure, Ptr).

\section{Simulated diseased respiratory conditions and active lung model settings}

Three diseased respiratory conditions were simulated: COPD, OHS and NMD. The patient's inspiratory effort was simulated by specifying a "muscular" pressure (Pmus) (equivalent to pleural pressure) in the ASL5000 [13]. In each breathing cycle, inspiration and expiration correspond to the contraction and relaxation phases of the inspiratory muscles, during which the Pmus can be represented by two exponential functions, respectively. These functions are characterized by the patient's breathing rate, the drop in muscular pressure at $100 \mathrm{~ms}$ (Pmus 0.1) reflecting patient's ventilatory drive [14] and the maximum Pmus decrease (Pmax) during the breathing cycle [15]. The values of inspiratory effort settings in the ASL5000 for the three simulated diseases were chosen according to published clinical values [16-20]. The ASL5000 settings corresponding to the simulated respiratory conditions are shown in Additional file 1 $[21,22]$.

\section{Simulated upper airways patency patterns}

For each diseased condition, we simulated open and closed UA by applying external pressures in the Starling resistor (Ps) of -8 and $+8 \mathrm{cmH} 2 \mathrm{O}$ respectively. The simulated airflow, the corresponding inspiratory muscular pressure and tidal volume of the three diseased conditions with open and closed UA are shown in Additional file 1.

\section{Tested devices and ventilatory settings}

Three life support home ventilators were evaluated in the study: Astral ${ }^{\text {mi }} 150$ (Resmed, NorthRyde, Australia; software version SX544-0301; denoted as A150), Trilogy $^{\text {ma }} 100$ (Philips Respironics, Monroeville, USA; software version 14.1.02; denoted as T100) and $\mathrm{Vivo}^{\mathrm{mm}} 60$

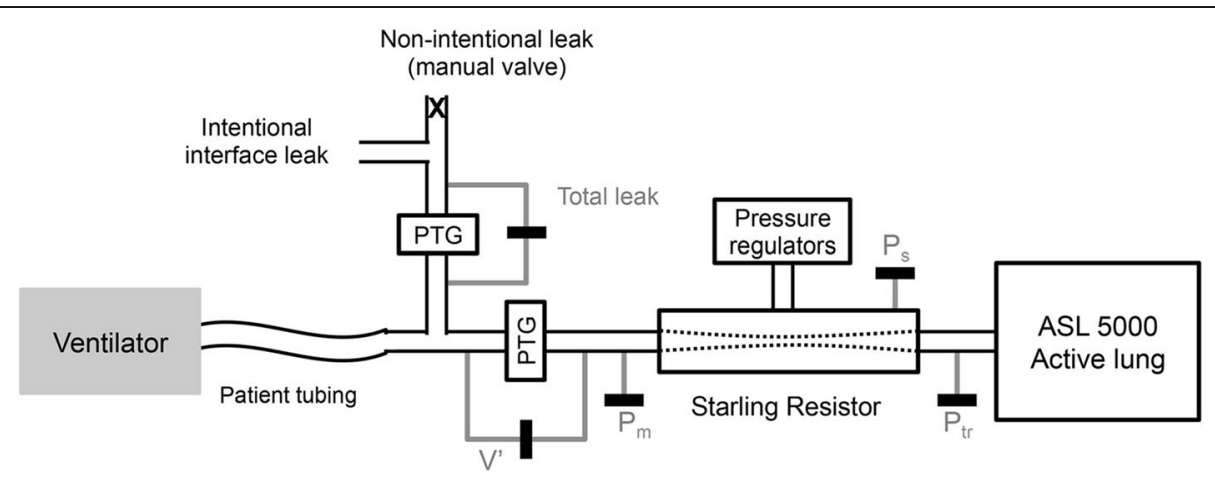

Fig. 1 The principle of the bench model. PTG: pneumotachograph; V': mask flow; Pm: Mask pressure; Ptr: tracheal pressure; Ps: pressure in the Starling resistor 
(Breas, Mölnlycke, Sweden; firmware version 3.05; denoted as V60). These devices were able to provide pressure-targeted ventilation with single-limb circuit configuration including an intentional leak. Because inspiratory trigger sensitivity was graduated in different units in each device, these settings were first comparatively evaluated with ASL5000 to establish equivalent sensitivities among devices. The details of methods and results are provided in Additional file 2.

Besides, other ventilatory settings such as rise time and I:E cycling were also comparatively evaluated and the corresponding comparisons are available in Additional file 2. Table 1 shows the chosen ventilatory settings for the current study.

\section{Patient-ventilator asynchrony: ineffective efforts and autotriggering}

PVA is defined as the mismatching between neural and mechanical inspiratory time [23-25]. Phase asynchronies, characterized by a mismatch between the start or the end of the patient's and the ventilator's inspiratory time are the most common asynchronies. They may occur at two periods: during inspiratory triggering, in situations in which there is a mismatch between patient inspiratory effort and ventilator triggering (i.e.: ineffective inspiratory effort, double triggering or autotriggering) or during cycling from inspiration to expiration, when ventilator cycling does not coincide with the end of patient effort (i.e.: premature or delayed cycling) [26].

In the current study, PVA during the triggering phase was characterized as a mismatch between the simulated inspiratory effort and the occurrence of the ventilator assisted cycle, which is reflected by whether or not a ventilatory cycle occurs following a decrease in Pmus [23-25]. Thus, an ineffective effort was defined as a simulated inspiratory effort that was not followed by an assisted cycle (i.e., delay longer than the inspiratory phase) and an autotriggered cycle as a non-backup cycle that was delivered by the ventilator during the expiratory phase without a prior Pmus decrease (Fig. 2). As the backup rate was set at $7 \mathrm{bpm}$, an autotriggered cycle

Table 1 Applied ventilator settings

\begin{tabular}{llll}
\hline & A150 & T100 & V60 \\
\hline Mode & ST & ST & Support \\
EPAP-IPAP $\left(\mathrm{CmH}_{2} \mathrm{O}\right)$ & $5-15$ & $5-15$ & $5-15$ \\
Backup respiratory rate (bpm) & 7 & 7 & 7 \\
l:E cycling & High & $40 \%$ & 5 (a.u.) \\
Rising time & 200 (ms) & 1 (a.u.) & 2 (a.u.) \\
Ti (s) & $0.8-1.5$ & 1.5 & $0.8-1.5$
\end{tabular}

ST spontaneous triggered pressure support mode with backup rate, EPAP expiatory positive airway pressure, IPAP inspiratory positive airway pressure, $T i$ inspiratory time, bpm breaths per minute. A150 Astral ${ }^{\mathrm{TM}}$ 150, T100 Trilogy $^{\mathrm{TM}} 100$ V60 Vivo $^{\mathrm{TM}} 60$, a.u. arbitrary unit could occur within $8.6 \mathrm{~s}$ after the beginning of the previous cycle.

The asynchrony level (AL) was defined as the ratio of the number of asynchrony events over the sum of the numbers of ventilatory cycles and ineffective efforts [26]. Therefore, the PVA included both ineffective efforts and autotriggered cycles.

\section{Protocol}

The ventilator was connected to the calibrated leak port built in the bench model through a $1.8-\mathrm{m}$ long and 22$\mathrm{mm}$ diameter tubing. The principal endpoint was to find for each ventilator the "critical leak" level at which the asynchrony appeared in COPD, OHS and NMD conditions with either open or closed UAs. The AL was calculated for each 5-min period at different leak levels. The "critical leak" was defined as the median value of the highest total leak level (intentional + unintentional leaks) during 5 min at which the AL remained lower than $25 \%$.

Figure 3 shows the algorithm used in the protocol for finding the "critical leak". Each test started with the intermediate inspiratory trigger setting of ventilator ("Medium" for A150, "5" for T100 and V60) since these settings were graduated in different units in each device and did not correspond to each other (Additional file 2). The total leak started with the minimum level, i.e., intentional leak with closed valve that simulated the unintentional leak (Fig. 3). At the end of each 5-min period, if the AL was lower than $25 \%$, the total leak would be manually increased in a stepwise manner by regulating the total end-expiratory leak in the following order: $30,40,45,50$ and $55 \mathrm{~L} / \mathrm{min}$; otherwise, the last leak level was maintained while the inspiratory trigger was adjusted in a stepwise manner to decrease the PVA events as follows: increase the trigger sensitivity if predominant events (>50\%) were ineffective efforts, or decrease the trigger sensitivity if predominant events (> $50 \%)$ were autotriggered cycles. This procedure was repeated until the critical leak was reached. An "effective adjustment" was defined as a manual adjustment of trigger sensitivity that decreased AL below 25\%. In each test, the inspiratory trigger settings that corresponded to the critical leak were noted and the number of repetitions necessary to obtain the final result was noted. In addition, leak values were compared between device reports and that measured on the bench. Details are presented in Additional file 3.

\section{Data analysis}

The following data were recorded with a sampling frequency of $20 \mathrm{~Hz}$ : mask airflow $\left(\mathrm{V}^{\prime}\right)$, mask pressure $(\mathrm{Pm})$, tracheal pressure (Ptr), pressure in the Starling resistor (Ps) and total leak. The muscular pressure (Pmus) was exported from the ASL5000 software 


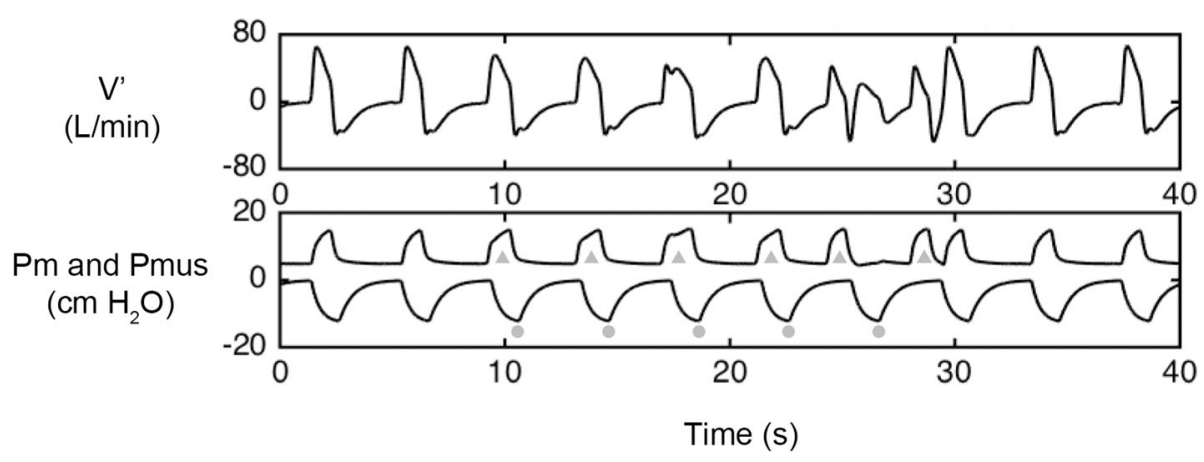

Fig. 2 Example of patient-ventilator asynchrony events. V': mask airflow; Pm: mask pressure; Pmus: inspiratory muscular pressure. An ineffective effort was defined as a simulated inspiratory effort that was not followed by an assisted pressure support in Pm (dot); An autotriggered cycle was defined as a non-backup ventilatory cycle that was delivered by the ventilator during the expiratory phase without a prior Pmus

decrease (triangle)

(IngMar Medical, Pittsburgh, USA) and was synchronized to the bench-recorded data for further analyses. For each 5-min period during which the inspiratory trigger and total leak level were unchanged, the number of autotriggered cycles and ineffective efforts were calculated by analyzing the Pm and Pmus curves. As leak flow follows the airway pressure, the distribution of leak values relates to that of the airway pressure. High leaks occurred at inspiratory phases when pressure support was applied; during the rest of time, a constant lower leak corresponding to the expiratory pressure persisted (i.e., the expiratory leak). The leak values during ventilatory cycles were thus not normally distributed. Consequently, the "critical leak" was presented as median (range, min-max values) of the 5 -min period. Comparisons of "critical leaks" between devices for each diseased respiratory and UA conditions were performed using Kruskal-Wallis tests. Statistical analyses were performed with MedCalc (MedCalc Software, Mariakerke, Belgium).

\section{Results}

Figure 4 and Additional file 4 show, respectively, the "critical leak" and the corresponding inspiratory trigger

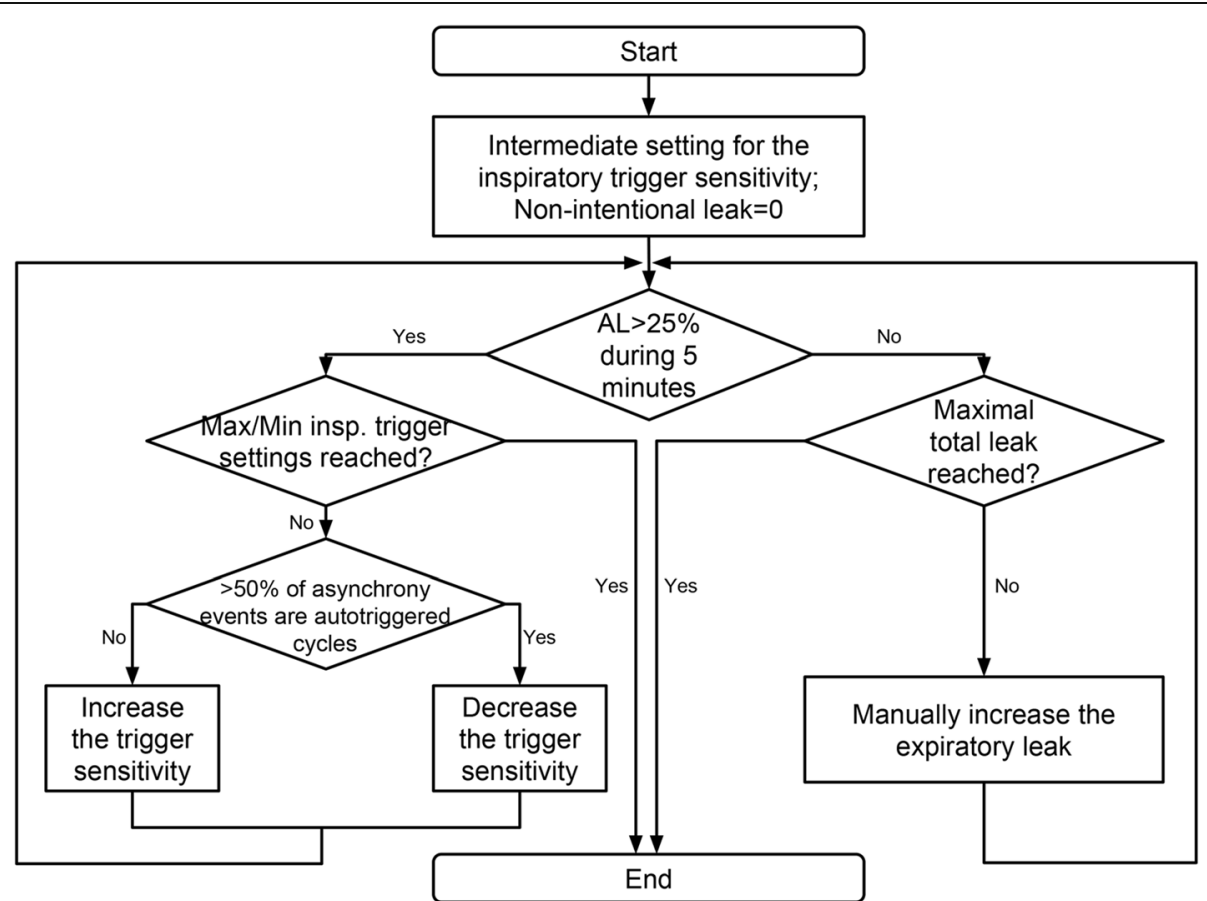

Fig. 3 The protocol for finding the « critical leak» of ventilators. AL: asynchrony level (ratio of the number of asynchrony events over the sum of the numbers of ventilatory cycles and ineffective efforts) 


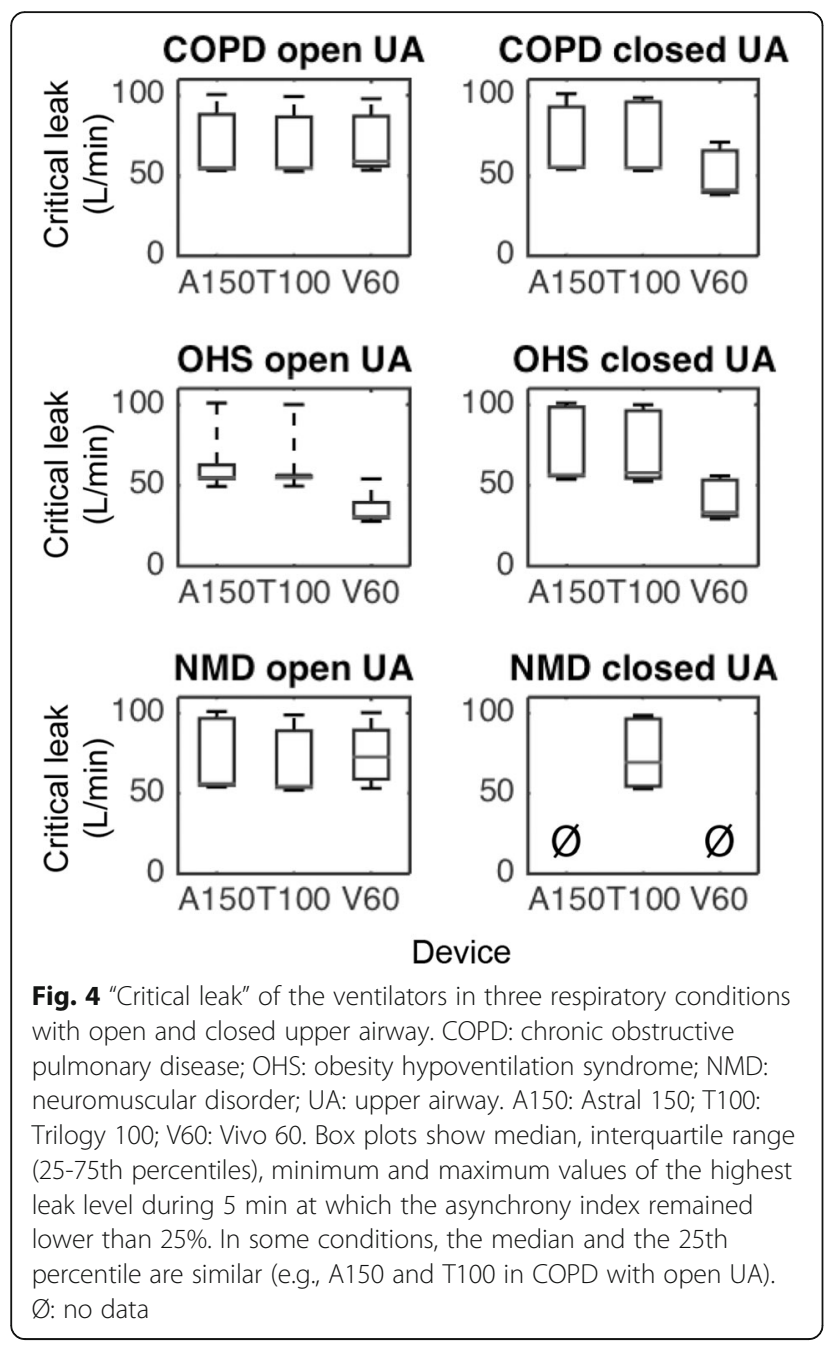

settings of the three home ventilators according to the simulated respiratory diseases.

With open UAs, all ventilators were able to avoid PVA in any lung conditions with critical leaks up to $31 \mathrm{~L} /$ min, and even up to $55 \mathrm{~L} / \mathrm{min}$ for some devices (Fig. 4). In OHS situation, the inspiratory trigger sensitivity of V60 was manually adjusted 4 times to most insensitive (setting 9) while autotriggered cycles still persisted when leak exceeded $31 \mathrm{~L} / \mathrm{min}$ (Fig. 5). Under other conditions, all ventilators were able to avoid both ineffective efforts and autotriggered cycles regardless the level of leak (Fig. 5, also see Additional file 4).

With closed UAs the results were heterogeneous between the simulated respiratory conditions and between devices. In COPD and OHS conditions, all ventilators were able to avoid PVA although the median critical leak was lower for V60 (41 and $33 \mathrm{~L} / \mathrm{min}$ for COPD and OHS, respectively) compared with A150 and T100 (higher than $55 \mathrm{~L} / \mathrm{min}$ each; see Figs. 4 and 5). In NMD condition, only T100 reached a critical leak of $69 \mathrm{~L} / \mathrm{min}$ (Fig. 4) while the other two ventilators failed to detect the inspiratory efforts even in the case of total leaks as low as $17 \mathrm{~L} / \mathrm{min}$ with the most sensitive inspiratory trigger settings ("very high" for A150 and "1" for V60; see Figs. 4 and 5). An example of the patient-ventilator asynchronies with closed UAs is given in Additional file 5. With closed UAs, the inspiratory trigger was changed 22 times in total in 9 PVA situations (all ventilators in all lung conditions), in which 5 PVA situations were alleviated $(\mathrm{AL}<25 \%)$ in the end after regulating the trigger sensitivity.

Results of comparison of leaks between device reports and bench-measured values are presented in Additional file 3.

\section{Discussion}

The goal of the current study was to evaluate the combined effects of leaks, mechanical properties of the respiratory system and UA patency on patient-ventilator synchrony during the triggering phase. We comparatively assessed on a previously reported bench model [12], the highest leak level tolerated without inducing significant PVA ("critical leak") in three home ventilators subjected to three simulated diseased respiratory conditions such as COPD, OHS and NMD. Each disease was simulated with both open and closed UAs. In addition, total leak values in the device reports were compared to the bench-measured values. The main findings were as follows: 1) with open UAs, all the ventilators were able to avoid PVA up to a $31 \mathrm{~L} / \mathrm{min}$ leak and even up to $55 \mathrm{~L} / \mathrm{min}$ in some cases. 2) UA closure and respiratory diseases, especially OHS, dramatically influenced PVA. With closed UAs, the critical leaks of A150 and T100 remained higher than $55 \mathrm{~L} / \mathrm{min}$ in COPD and OHS, while for V60 these values decreased to 41 and $33 \mathrm{~L} /$ min, respectively. In NMD with closed UAs, only T100 reached a high critical leak of $69 \mathrm{~L} / \mathrm{min}$. 3) Inspiratory trigger sensitivity change is often necessary to avoid PVA.

Since leak is the first cause of failure of NIV, inspiratory trigger performance of ventilators need to be tested in presence of leak. We confirm here the improvement of the inspiratory triggers in new ventilators: without UAs closure, all the ventilators were able to avoid PVA up to $30 \mathrm{~L} / \mathrm{min}$ leaks, and even up to $55 \mathrm{~L} / \mathrm{min}$ sometimes. These new flow-based triggering algorithms are more sensitive than classical flow triggering, and allow adjusting trigger sensitivity in presence of leak and, in this way, help to reduce ineffective efforts and autocycling. Using an "NIV algorithm" in ICU ventilators has been shown to significantly decrease the impact of leaks on the occurrence of patient-ventilator trigger asynchrony in some clinical cases [23]. But to our knowledge, our study is the first to systematically and comparatively evaluate the effect of unintentional leaks 


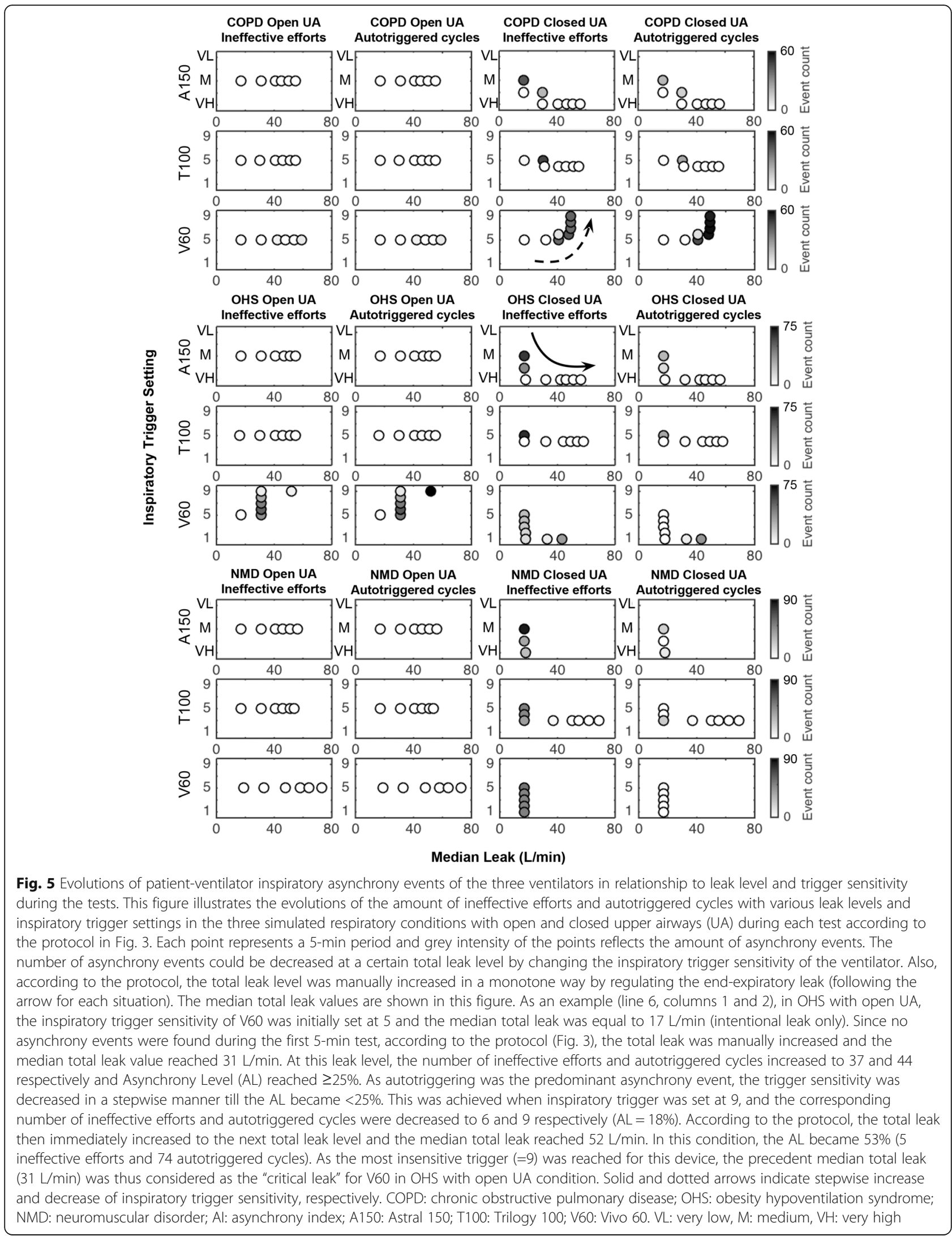


on triggering function in different ventilators using sophisticated flow-based trigger algorithms.

It has been stated that an unintentional leak lower than $24 \mathrm{~L} / \mathrm{min}$ is clinically tolerable in most cases [27]. Our study shows that the adverse effects of leaks during the triggering phase vary according not only to the absolute leak level but also to the mechanical properties of the lung and UA patency. The type of ventilator also affects the negative effect of leaks on the occurrence of PVA. We show that the threshold value of clinically "tolerable" leaks must be determined for each ventilator and that these thresholds differ between different devices and/or clinical situations. We also show that, without UA obstruction, all ventilators performed well with leaks lower than $30 \mathrm{~L} / \mathrm{min}$ which are clinically tolerable [27].

Clinical situations may also lead to PVA: UA obstruction and respiratory diseases. Most studies evaluating ventilator performance tested them by applying different respiratory mechanics conditions and leaks levels [2831]. The originality of our lung model is the use of a Starling resistor simulating UA closure on a bench model that takes into account the pressure responses of tested devices in a "closed loop" setting (i.e. with a direct interaction of the ventilator to the bench condition). To our knowledge, no study has evaluated so far the influence of varying UA resistance on leak-induced patientventilator trigger asynchronies.

Intermittent obstruction of UA during sleep is common under NIV and may be related to obstructive events at the oropharyngeal level (obstructive sleep apnea-hypopnea) because of insufficient expiratory positive airway pressure (EPAP) during sleep, in subjects with increased UA collapsibility. Currently, obstructive sleep apnea-hypopnea may be observed in all patients receiving NIV, in particular those with OHS. As assisted ventilator cycles are triggered either by the pressure drop in the proximal airway or by the detection of flow variation in the presence of a continuous flow washing out the circuit during expiration, this reduction in UA patency may reduce the capacity of the ventilator to detect patient inspiratory effort. Therefore, the EPAP should be increased prior to any changes in inspiratory trigger sensitivity when obstructive respiratory events occur, especially for low breathing efforts such as the case of neuromuscular diseases.

Finally, adjustable inspiratory trigger is an option presently available in most home ventilators and our results suggest that manual adjustment of the inspiratory trigger sensitivity allows a decrease of PVA. After verifying leak and EPAP levels, the inspiratory trigger sensitivity can be adjusted depending on the type of asynchrony events as described in our protocol (Fig. 3). However, in routine, this can be difficult as patient's conditions change daily. An automatic adaptation of the trigger sensitivity, taking into account the presence of leaks but also of PVA could be useful in the future.

Currently, home ventilators allow leaks to be estimated, but the performance of their algorithms is variable [32]. Also, intentional leaks are variable and proportional to the level of pressure. They differ between interfaces, and are included in the estimation of leaks in most algorithms [33]. Our study confirms data of Contal et al. concerning the differences in the accuracy of several devices in estimating unintentional leaks. In our study, only one device (T100) accurately estimated leak values at all tested levels as compared with bench-measured values. Moreover, in one of the devices (V60) this bias enlarged as the leak level increased. Thus, our study suggests that detecting and knowing accurately the magnitude of unintentional leaks and particularly their impact on the quality of ventilation is of major importance when monitoring NIV. Also, the methods of calculating leaks applied by ventilators remain to be standardized, especially those concerning the timing within a breathing cycle at which leaks are measured.

Some limitations may be made with regard to our study. First, we used a respiratory bench model. Even if advantages of this approach are evident (standardization of mechanical characteristics, repeatability, ability to study a broad range of controlled situations), this is certainly a simplified representation of a patient's complex inspiratory effort profile and pulmonary mechanics. Next, in the clinical setting this profile may vary over time. As an example, long term NIV is mainly applied during sleep, a physiological state characterized by variations in ventilatory drive, respiratory mechanics, UA patency and respiratory muscle recruitment during different sleep stages and changes in body position. Last, there are inherent limitations to bench testing per se: we applied a step-by-step leak increment. Indeed, the model does not integrate the inherent variability of unintentional leaks when the amount of leaks may rapidly vary in few cycles or even during the same cycle. Taking into account these limitations, there is a body of literature that suggests that bench testing is, however, an important and relevant component of the assessment of home ventilators. We evaluated three ventilators and applied our model to three simulated diseased respiratory conditions that may not be representative neither of the broad spectrum of available home ventilators nor reflecting the large range of clinical situations.

\section{Conclusions}

In conclusion, we demonstrated that performances of modern home ventilators are able to avoid PVA at much higher level of leaks than previously published. Unfortunately, in case of abnormal mechanical properties of the 
respiratory system or closed UA this function is strongly compromised with very heterogeneous results and manual changes in trigger sensitivity are often necessary to avoid PVA. Difference in coping with leaks and in the capacity of estimating leaks may account for betweendevice differences in effectiveness of NIV. As detecting and knowing unintentional leaks are of major importance when monitoring NIV, physicians must be aware of these discrepancies and must be able to set the sensitivity of the inspiratory triggers although this is usually difficult. Besides, in case of closed UA, the first setting to adjust should be the EPAP rather than the inspiratory trigger. Automatic detection of leaks and adaptation of triggering sensitivity by the devices is desirable, but further efforts are needed to improve leak estimation and ventilator performance during leaks and to clarify how leaks must be detected and understood: when are they "acceptable" and at what point do they become a problem.

\section{Additional files}

Additional file 1: Active lung model settings and simulated breathing patterns. Details of simulation of diseased breathing patterns. (PDF $144 \mathrm{~kb})$

Additional file 2: Study of equivalent ventilatory settings. Following ventilatory settings were analyzed: inspiratory trigger sensitivity, pressure rise time and I:E cycling. (PDF $265 \mathrm{~kb}$ )

Additional file 3: Comparison of leaks between device reports and bench-measured values. Protocol and results of comparing the leak values between device reports and bench measures. (PDF $232 \mathrm{~kb}$ )

Additional file 4: Inspiratory trigger settings corresponding to "critical leak" values. Table of the inspiratory trigger settings of the three ventilators corresponding to the "critical leaks" according to the protocol in Fig. 3. (PDF 80 kb)

Additional file 5: Example of patient-ventilator asynchronies with closed upper airways. Detailed curves of airway flow, pressures and leaks illustrating observed patient-ventilator asynchronies. (PDF $5414 \mathrm{~kb}$ )

\section{Abbreviations}

AL: Asynchrony level; COPD: Chronic obstructive pulmonary disease; EPAP: Expiratory positive airway pressure; NIV: Non-invasive ventilation; NMD: Neuromuscular disorders; OHS: Obesity hypoventilation;

Pmax: Maximum Pmus decrease; Pmus: Muscular pressure; Ps: Pressure in the Starling resistor; Ptr: Tracheal pressure; PVA: Patient-Ventilator Asynchrony; UA: Upper Airway

\section{Acknowledgements}

Not applicable.

\section{Funding}

No funding was received for this study.

\section{Availability of data and materials}

The datasets used and/or analysed during the current study are available from the corresponding author on reasonable request.

\section{Authors' contributions}

$K Z, C R, J G B, P E$ and GR wrote the initial draft. KZ executed the experiments and analysed the data. All authors made substantial contributions to the conception or design of the work, or the acquisition, analysis or interpretation of data for the work, revised the manuscript for important intellectual content, and agreed to be accountable for all aspects of the work. All authors have read and approved the final version of this manuscript.

Ethics approval and consent to participate

Not applicable.

\section{Consent for publication}

Not applicable.

\section{Competing interests}

The authors declare that they have no competing interests.

\section{Publisher's Note}

Springer Nature remains neutral with regard to jurisdictional claims in published maps and institutional affiliations.

\section{Author details}

${ }^{1}$ Centre Explor, Air Liquide Healthcare, 28 rue d'Arcueil, 94250 Gentilly, France. ${ }^{2}$ Service de Pneumologie et Soins Intensifs Respiratoires, Centre Hospitalier Universitaire Dijon Bourgogne, 14 rue Paul Gaffarel, F-21079 Dijon, France. ${ }^{3}$ Sorbonne Universités, UPMC Univ Paris 6, INSERM, UMRS1158 Neurophysiologie Respiratoire Expérimentale et Clinique, Paris, France. ${ }^{4}$ Service de Pneumologie et Réanimation Médicale (Département "R3S"), AP-HP, Groupe Hospitalier Pitié-Salpêtrière Charles Foix, F-75013 Paris, France. ${ }^{5}$ Service des Explorations Fonctionnelles Multidisciplinaires, AP-HP, Hôpital Antoine-Béclère, 157 rue de la Porte de Trivaux, 92140 Clamart, France.

Received: 25 May 2017 Accepted: 10 November 2017

Published online: 21 November 2017

\section{References}

1. Goldberg A. Clinical indications for noninvasive positive pressure ventilation in chronic respiratory failure due to restrictive lung disease, COPD, and nocturnal hypoventilation-a consensus conference report. Chest. 1999;116: 521-34.

2. Lloyd-Owen SJ, Donaldson GC, Ambrosino N, Escarabill J, Farre R, Fauroux B, et al. Patterns of home mechanical ventilation use in Europe: results from the Eurovent survey. Eur Respir J. 2005;25:1025-31.

3. Vogelmeier CF, Criner GJ, Martinez FJ, Anzueto A, Barnes PJ, Bourbeau J, et al. Global strategy for the diagnosis, management, and prevention of chronic obstructive lung disease 2017 report: GOLD executive summary. Eur Respir J. 2017:49:1700214.

4. Teschler H, Stampa J, Ragette R, Konietzko N, Berthon-Jones M. Effect of mouth leak on effectiveness of nasal bilevel ventilatory assistance and sleep architecture. Eur Respir J. 1999;14:1251-7.

5. Meyer TJ, Pressman MR, Benditt J, McCool FD, Millman RP, Natarajan R, et al. Air leaking through the mouth during nocturnal nasal ventilation: effect on sleep quality. Sleep. 1997;20:561-9.

6. Rabec C, Rodenstein D, Leger P, Rouault S, Perrin C, Gonzalez-Bermejo J. Ventilator modes and settings during non-invasive ventilation: effects on respiratory events and implications for their identification. Thorax. 2011;66:170-8.

7. Fanfulla F, Delmastro M, Berardinelli A, Lupo ND, Nava S. Effects of different ventilator settings on sleep and Inspiratory effort in patients with neuromuscular disease. Am J Respir Crit Care Med. 2005;172:619-24.

8. Fanfulla F, Taurino AE, Lupo ND, Trentin R, D'Ambrosio C, Nava S. Effect of sleep on patient/ventilator asynchrony in patients undergoing chronic noninvasive mechanical ventilation. Respir Med. 2007;101:1702-7.

9. Crescimanno G, Canino M, Marrone O. Asynchronies and sleep disruption in neuromuscular patients under home noninvasive ventilation. Respir Med. 2012;106:1478-85

10. Sancho J, Servera E, Morelot-Panzini C, Salachas F, Similowski T, GonzalezBermejo J. Non-invasive ventilation effectiveness and the effect of ventilatory mode on survival in ALS patients. Amyotroph Lateral Scler Front Degener. 2014;15:55-61.

11. Georges M, Attali V, Golmard JL, Morélot-Panzini C, Crevier-Buchman L, Collet J-M, et al. Reduced survival in patients with ALS with upper airway obstructive events on non-invasive ventilation. J Neurol Neurosurg Psychiatry. 2016;87:1045-50. 
12. Zhu K, Roisman G, Aouf S, Escourrou P. All APAPs are not equivalent for the treatment of sleep disordered breathing: a bench evaluation of eleven commercially available devices. J Clin Sleep Med. 2015;11:725-34.

13. Chatburn RL. Which ventilators and modes can be used to deliver noninvasive ventilation? Respir Care. 2009;54:85-101.

14. American Thoracic Society/European Respiratory Society. ATS/ERS statement on respiratory muscle testing. Am J Respir Crit Care Med. 2002;166:518-624.

15. Fresnel $E$, Muir J-F, Letellier C. Realistic human muscle pressure for driving a mechanical lung. EPJ Nonlinear Biomed Phys. 2014;2:1-18.

16. Murciano D, Aubier M, Bussi S, Derenne J-P, Pariente R, Milic-Emili J. Comparison of esophageal, tracheal, and mouth occlusion pressure in patients with chronic obstructive pulmonary disease during acute respiratory failure. Am Rev Respir Dis. 1982;126:837-41.

17. Montes de Oca M, Celli BR. Mouth occlusion pressure, $\mathrm{CO} 2$ response and hypercapnia in severe chronic obstructive pulmonary disease. Eur Respir J. 1998;12:666-71.

18. Budweiser S, Jörres RA, Criée C-P, Langer V, Heinemann F, Hitzl AP, et al. Prognostic value of mouth occlusion pressure in patients with chronic ventilatory failure. Respir Med. 2007;101:2343-51.

19. Heinemann F, Budweiser S, Dobroschke J, Pfeifer M. Non-invasive positive pressure ventilation improves lung volumes in the obesity hypoventilation syndrome. Respir Med. 2007;101:1229-35.

20. Baydur A. Respiratory muscle strength and control of ventilation in patients with neuromuscular disease. Chest. 1991;99:330-8.

21. Olivieri C, Costa R, Conti G, Navalesi P. Bench studies evaluating devices for non-invasive ventilation: critical analysis and future perspectives. Intensive Care Med. 2011;38:160-7.

22. Oto J, Chenelle CT, Marchese AD, Kacmarek RM. A comparison of leak compensation in acute care ventilators during non-invasive and invasive ventilation; a lung model study. Respir Care. 2013;58(12):2027-37.

23. Carteaux G, Lyazidi A, Cordoba-Izquierdo A, Vignaux L, Jolliet P, Thille AW, et al. Patient-ventilator asynchrony during noninvasive ventilation: a bench and clinical study. Chest. 2012;142:367-76.

24. Guo YF, Sforza E, Janssens JP. Respiratory patterns during sleep in obesityhypoventilation patients treated with nocturnal pressure support: a preliminary report. Chest. 2007;131:1090-9.

25. Ramsay M, Mandal S, Suh E-S, Steier J, Douiri A, Murphy PB, et al. Parasternal electromyography to determine the relationship between patient-ventilator asynchrony and nocturnal gas exchange during home mechanical ventilation set-up. Thorax. 2015;70:946-52.

26. Thille AW, Rodriguez P, Cabello B, Lellouche F, Brochard L. Patient-ventilator asynchrony during assisted mechanical ventilation. Intensive Care Med. 2006;32:1515-22.

27. Teschler H. Monitoring of the home mechanical ventilated patient. In: Muir J-F, Ambrosino N, Simonds AK, eds. Noninvasive Ventilation. Eur Respir Mon. 2001;16:274-80.

28. Vignaux L, Vargas F, Roeseler J, Tassaux D, Thille AW, Kossowsky MP, et al. Patient-ventilator asynchrony during non-invasive ventilation for acute respiratory failure: a multicenter study. Intensive Care Med. 2009;35:840-6.

29. Khirani S, Louis B, Leroux K, Delord V, Fauroux B, Lofaso F. Harms of unintentional leaks during volume targeted pressure support ventilation. Respir Med. 2013;107:1021-9.

30. Luján M, Sogo A, Pomares X, Monsó E, Sales B, Blanch L. Effect of leak and breathing pattern on the accuracy of tidal volume estimation by commercial home ventilators: a bench study. Respir Care. 2013;58:770-7.

31. Sogo $A$, Montanyà J, Monsó E, Blanch L, Pomares X, Lujàn M. Effect of dynamic random leaks on the monitoring accuracy of home mechanical ventilators: a bench study. BMC Pulm Med. 2013;13:75.

32. Contal $\mathrm{O}$, Vignaux L, Combescure C, Pepin J-L, Jolliet P, Janssens J-P. Monitoring of noninvasive ventilation by built-in software of home bilevel ventilators: a bench study. Chest. 2012;141:469-76.

33. Rabec CA, Reybet-Degat O, Bonniaud P, Fanton A, Camus P. Leak monitoring in noninvasive ventilation. Arch Bronconeumol Engl Ed. 2004:40:508-17.

\section{Submit your next manuscript to BioMed Central and we will help you at every step:}

- We accept pre-submission inquiries

- Our selector tool helps you to find the most relevant journal

- We provide round the clock customer support

- Convenient online submission

- Thorough peer review

- Inclusion in PubMed and all major indexing services

- Maximum visibility for your research

Submit your manuscript at www.biomedcentral.com/submit

) Biomed Central 